\title{
Análise da influência dos diferentes tempos de polimerização por luz incandescente sobre a resistência ao desgaste abrasivo de resinas compostas
}

CRUZ, C.E I. SANTANA, L R. I. BIANCHI, E.C I. DE AGUIAR, P R. I. SILVA JUNIOR, C.E. CATAI, R.E. II; DE FREITAS, C.A. ${ }^{\text {III }}$; SALGADO, M.H. ' ; FREITAS, M.F.A. ${ }^{\text {III }}$, ULHOA, M.P.M. ${ }^{\text {IV }}$

\author{
${ }^{\mathrm{I}}$ Universidade Estadual Paulista - UNESP - Campus de Bauru. Dept ${ }^{\mathrm{o}}$. de Eng ${ }^{\mathrm{a}}$. Mecânica \\ Av. Luiz Edmundo Carrijo Coube s/n., Caixa Postal 473, CEP 17033-360, Bauru, SP, Brasil. \\ e-mail: cedc@feb.unesp.br, 1rssantana@ffeb.unesp.br, bianchi@,feb.unesp.br, \\ aguiarpr@feb.unesp.br, cesilvaj@gmail.com, henri@feb.unesp.br \\ ${ }^{\text {II } U n i v e r s i d a d e ~ E s t a d u a l ~ d e ~ C a m p i n a s ~-~ U N I C A M P ~-~ F a c u l d a d e ~ d e ~ E n g ~}{ }^{\text {a }}$. Mecânica - DEF/FEM \\ Caixa Postal 6122, CEP 13083-970, Campinas, SP, Brasil. \\ e-mail: rcatai@,fem.unicamp.br

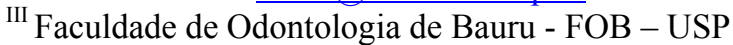 \\ Al. Octávio Pinheiro Brisola, 9-75, CEP 17012-901, Bauru, SP, Brasil. \\ e-mail: cfreitas@,fob.usp.br, mfafreitas@yahoo.com.br \\ ${ }^{\text {IV }}$ Universidade Estadual Paulista - UNESP - Campus de Bauru. Faculdade de Ciências \\ Av. Luiz Edmundo Carrijo Coube s/n., Caixa Postal 473, CEP 17033-360, Bauru, SP, Brasil. \\ e-mail: michelepaoline@yahoo.com.br
}

\section{RESUMO}

No mercado atual a qualidade é necessária na fabricação dos produtos. No mercado odontológico, as resinas compostas são cada vez mais utilizadas para substituir materiais tradicionais, como amalgama, nas restaurações estéticas e funcionais, apresentando boa estética e funcionalidade. A composição química destes materiais é variável e os resultados das reações químicas do processo de polimerização são bastante desconhecidos. O objetivo deste trabalho era medir a resistência do desgaste abrasivo de seis resinas compostas vendidas no mercado brasileiro polimerizadas com tempos de 10, 20 e 40 segundos. O método do disco retificado modificado para aplicações em odontologia foi usado para tornar esta proposta viável. Neste banco de ensaios um disco dinâmico, revestido por porcelana, desgasta um disco estático revestido do tipo de resina que se pretende avaliar, que foi polimerizada durante um tempo pré-estabelecido. Com a determinação do desgaste de material, num determinado tempo, determinava-se a agressividade (capacidade de um material para desgastar outro material) do disco dinâmico sobre o disco estático. Utilizando-se para isso um programa computacional (LabView). Posteriormente era feita a regressão linear dos valores obtidos e determinado o coeficiente angular, utilizado no cálculo de agressividade. Valores elevados de agressividade indicam uma maior agressão do disco dinâmico contra o disco estático. Os resultados mostraram um comportamento variável para cada material que foi polimerizado nos diferentes tempos de polimerização utilizado, mostrando que o método para fazer estudos de desgaste é prático e rápido para testar materiais novos antes da sua utilização.

Palavras chaves: Resinas compostas, desgaste abrasivo, tempo de fotopolimerização.

\section{Analysis of the influence of the different times of polymerization by incandescent light in composite resins by evaluation of its abrasive wear ABSTRACT}

In the current market an increasing quality is demanded to manufacture products. At the odontologic market the composed resins are becoming more and more used to replace old materials, like amalgamates in the aesthetic and functional restorations, since they present a better appearance and easier manuscript. The chemical composition of these materials varies and the results of the chemical reactions of polymerization processes are quite unknown. The aim of this work was to measure the resistance of the abrasive wear of six composed resins sold in Brazilian market when displayed to polymerization periods of 10, 20 and 40 seconds. The grinding disc method appropriated for odontology was used to make this process possible. For 
this a bank of assays in which a porcelain covered dynamic disc consumes a static disc recovered by the polymerized resin is used to demonstrate the method in a pre-established time. The abrasive wear was determined by the calculation of the aggressiveness (capacity of a material to consume another). This aggressiveness is determined by an initial graph of displacement for time, through the computational method (LabView program). After they have passed through a linear regression the results give as a possibility the angular coefficient, considered a parameter at the calculation of aggressiveness. Higher values of aggressiveness which means a higher aggression suffered by the resin disc indicate worse resistance to the abrasive consuming of this resin in relation to porcelain. The results showed a variable comportment for each material ahead the different polymerization time, which prove the importance in create a practical and fast analyses method to test new materials before their use.

Keywords: Composite resins, abrasive wear, time of photopolymerization.

\section{INTRODUÇÃO}

O homem está sempre em busca de melhores condições de vida. A valorização da estética pela sociedade atual é uma delas, resultando em avanços no desenvolvimento de novos materiais sintéticos na Odontologia. Dentro destes avanços as novas fórmulas de resinas compostas fotopolimerizáveis, as quais são amplamente utilizadas como materiais restauradores para dentes anteriores e posteriores, são as que mais se destacam, principalmente em substituição a amalgama.

Dentre os vários fatores que influenciam aos dentistas a utilizarem constantemente estas resinas, estão à facilidade no manuseio e uma grande quantidade de cores, possibilitando ficarem praticamente imperceptíveis após sua aplicação.

As resinas compostas foram escolhidas entre vários materiais de restauração dentária por apresentarem menor desgaste, maior facilidade de manuseio (são vendidas em tubos como os de uma pasta dental) e por possuírem uma grande gama de cores, possibilitando-se alcançar a semelhança com a coloração dos dentes humanos, tornando-se praticamente imperceptível após sua aplicação.

Para que esses fossem restaurados adequadamente, entre outras características, deveriam apresentar resistência ao desgaste tão alta quanto o esmalte dentário, o que não ocorre. Com os esforços gerados na mastigação e escovações dos dentes ocorre um desgaste que leva a uma substituição precoce da restauração.

A American Dental Association - ADA [1] relatou que ainda eram limitadas as informações de longo prazo do desempenho clínico das resinas compostas em dentes posteriores, o que impulsionou diversas pesquisas relativas à resistência desses materiais. Duas linhas de estudo são largamente utilizadas. O estudo clínico ou "in vivo" e o laboratorial ou "in vitro".

O fator limitante do uso das resinas compostas é a deterioração que ocorre quando estes materiais são utilizados onde estão sujeitos ao desgaste oclusal [14]. Esse tem sido analisado em um grande número de estudos clínicos, e vários tipos de testes têm sido desenvolvidos para analisá-lo nas resinas compostas e também em outros materiais restauradores. Uma análise confiável da resistência ao desgaste abrasivo deve auxiliar no desenvolvimento e melhora das resinas compostas, pois fornecerá uma forma de análise rápida destes produtos, sem a necessidade de aguardar os resultados dos demorados ensaios clínicos.

De acordo com a literatura, para a realização das análises do desgaste de resinas compostas são apresentados métodos clínicos ("in vivo") e métodos laboratoriais ("in vitro"). O método clínico in vivo consiste em realizar restaurações em um determinado número de pacientes e, após certo período de tempo, normalmente bastante longo, cerca de 2 a 6 anos, analisar o desgaste ocorrido na resina. Neste método, existe a falta de controle sobre variáveis importantes como a força empregada na mastigação, à dieta ou fatores biológicos (bucais), que limitam o estudo clínico e em conseqüência, o estudo do desgaste dos materiais dentários.

Já nos testes laboratoriais esses inconvenientes são minimizados. Dessa forma torna-se interessante realizar ensaios in vitro, que é um método que propõe ensaios laboratoriais e pode reduzir o tempo e o custo das análises com as resinas para avaliar seu comportamento, suas características e eficiência contra o desgaste abrasivo, entre outras variáveis, buscando resultados precisos, porém mais rápidos que os testes in vivo.

Diversos autores, entre eles Miranda et al. [2] e Wendt \& Leinfelder []], apresentaram trabalhos em que demonstravam um comportamento indevido das resinas como materiais restauradores para utilização em dentes posteriores, fato ligado ao seu alto desgaste oclusal e consecutiva perda de forma anatômica, em comparação a outros materiais como o amalgama. Além disso, Collins et al. [4] relatou, após oito anos de análise, a existência de falhas em restaurações com resinas de 2 a 3 vezes maior que as ocorridas no amalgama.

O outro método usado para avaliação do desgaste de resinas é o método clínico, ou de avaliações "in vitro". Este método consiste na construção de simuladores do desgaste sofrido pelas resinas na boca humana. 
As vantagens deste tipo de análise estão ligadas ao controle de variáveis, fato impossível de ser realizado nos estudos em seres humanos, tornando desta forma os dados mais confiáveis.

Contudo o maior problema apresentado pelo método consiste no seu baixo índice de correlação com os ensaios clínicos. Ressalta-se a importância do desenvolvimento de ambos os métodos, pois apenas desta forma pode haver uma evolução efetiva tendo em vista as razões já apresentadas.

Diversos equipamentos foram desenvolvidos a fim de simular o desgaste ocorrido na boca humana. Do estudo dos textos destacam-se os autores Mair et al. [5], Yap et al. [6], Condon \& Ferracani [7] e Momoi et al. [8].

A partir da análise de seus trabalhos verifica-se que o desgaste das resinas compostas convencionais é um processo onde há a remoção das partículas de material inorgânico e a matriz da resina é continuamente desgastada. Sob tensão e sob a ação abrasiva do alimento, ocorre à micro-abrasão da matriz, causando posteriormente a exposição das partículas inorgânicas e o conseqüente aumento da tensão.

$\mathrm{O}$ desgaste também é influenciado pela fadiga mecânica. O movimento dos dentes durante a mastigação, tensões cíclicas, juntamente com carregamentos e descarregamentos, podem iniciar e propagar micro trincas. Pequenas trincas ainda podem ser geradas por tensões elevadas entre a matriz e as partículas (cargas), causadas pela grande diferença entre seus coeficientes de dilatação.

Como fator atuante na aceleração do processo de deterioração ainda há a diferença entre os módulos de elasticidade da matriz e da carga. Observações das restaurações realizadas com estes tipos de resinas em superfícies oclusais revelaram que a perda de material é normalmente uniforme.

O material removido da superfície desgastada pode ser atribuído a diversos fatores como: erosão, corrosão, adesão, abrasão e impacto. O desgaste por erosão ocorre quando partículas sólidas ou líquidas colidem com a superfície do material. $\mathrm{O}$ desgaste por corrosão pode ser definido como uma forma química de desgaste resultante da interação do meio com a superfície. O desgaste por adesão ocorre quando material sólido desliza sobre a superfície de outro material, ou é pressionado contra ele, causando remoção de pequenas partículas da superfície. O desgaste abrasivo ocorre quando uma superfície dura e rugosa arranca partículas de um material de menor dureza. O desgaste por impacto é definido como sendo proveniente de choques repetitivos entre duas superfícies.

Não só o desgaste e a forma com que ele ocorre é parâmetro importante de análise. A polimerização da resina é um processo complexo e diversas são as variáveis que podem atuar para que a mesma seja realizada de maneira mais eficiente, como é mostrado por: Venhovem et al. [9], Vieira et al. [10], Abate et al. [11] e Halvorson et al. [12].

Para a confecção de corpos de provas para a realização de ensaios, as resinas compostas são polimerizadas com aparelhos de fotopolimerização. Kurachi et al. [15], em seu estudo, afirmam que com um maior desenvolvimento dos instrumentos de fotopolimerização baseados em LED, estes podem tornar-se dispositivos mais eficientes que as lâmpadas convencionais. Realizaram um estudo comparando durezas avaliadas em uma resina composta polimerizada por cinco dispositivos baseados em LED (diodo emissor de luz com uma unidade de polimerização convencional). Todas as amostras fotopolimerizadas pelos dispositivos baseados em LED mostraram valores inferiores de dureza quando comparados com a lâmpada halógena no tempo de fotopolimerização típico ( 40 s). Concluiu também que tempos de exposição longos ou uma camada de resina mais fina são necessários para conseguir valores razoáveis da dureza em aparelhos baseados em LED.

Tsai et al. [16] concluíram em seu trabalho que as luzes de polimerização LED disponíveis comercialmente usadas no estudo oferecem profundidade de polimerização e microdureza adequadas para espessuras de resinas que são recomendadas para uso no método de construção incremental $(2 \mathrm{~mm}$ ou menos). Para maiores espessuras de resinas, a desempenho das luzes de polimerização LED não é igual àquela das luzes de polimerização de quartzo tungstênio halógenas convencionais de alta intensidade.

Para se analisar a qualidade de resinas compostas, além dos ensaios de desgaste abrasivo, são efetuados ensaios de microdureza, entre outros. Ao realizar a análise in vitro de dureza, da contração de polimerização e da rugosidade de 29 resinas compostas, Carvalho Júnior [17] concluiu que todas as resinas mostraram aumento da dureza e da contração de polimerização, ao se compararem os valores das três características obtidos para a medição imediata com os valores obtidos após 168 horas.

O estudo dos trabalhos apresentados acima foi de essencial importância na determinação de alguns parâmetros deste trabalho, como será visto na parte de apresentação descrição do método.

\section{MATERIAIS E MÉTODOS}

O método utilizado para a avaliação do desgaste de resinas compostas baseou-se no estudo do comportamento da agressividade (capacidade de um material desgastar o outro) da superfície de restaurações dentárias confeccionadas com essas resinas. Para a determinação deste parâmetro foi reproduzido e adaptado o método do disco retificado desenvolvido por Coelho [13], originalmente utilizado para avaliar a 
agressividade de rebolos na retificação de peças de aço. Aperfeiçoado para a utilização em materiais dentários, este banco de ensaios consiste basicamente em manter um disco de porcelana girando acima de um disco revestido com resina.

Os corpos de prova, denominados aqui de discos, tem formato cilíndrico. O disco de porcelana ou disco agressor é feito em sua parte mais interna de uma liga de Níquel-Cromo e em sua periferia revestido por porcelana. O disco agredido de resina é confeccionado com aço dúctil (ABNT 1020) e revestido em todo seu perímetro circular com resina composta, a qual se deseja analisar.

Trata-se de um método diferenciado ao que tradicionalmente é feito em odontologia (onde se estuda o desgaste de resinas por escovação ou pelo acompanhamento do desgaste de resinas em dentes restaurados), sendo mais rápidos os ensaios e a obtenção dos resultados.

A descrição detalhada das etapas de fabricação dos discos será mostrada no item a seguir.

\subsection{Confecção dos Discos}

Anterior à realização dos ensaios foi feita à determinação de parâmetros de avaliação e a preparação dos corpos de prova, descrita a seguir.

\subsection{Disco Dinâmico}

O disco dinâmico, ou disco agressor é constituído por três materiais. A parte externa é feita de porcelana, material escolhido para simular o material dos dentes, sendo que o mesmo é usado na confecção de próteses de substituição dentária. A aderência da porcelana, na parte intermediária é feita com uma liga metálica específica para este uso.

A fabricação desta parte consistiu na fundição de um tarugo de liga metálica de níquel-cromo (NiCr), comercializada com o nome de Durabond (nome fantasia). Depois de fundido, o tarugo foi torneado de modo a adquirir dimensões de: espessura de $16 \mathrm{~mm}$, diâmetro interno de $15 \mathrm{~mm}$ e diâmetro externo de 18 $\mathrm{mm}$. Para melhor acabamento, depois de torneado, o tarugo foi retificado.

O tarugo, de agora em diante identificado por anel, recebeu uma camada do opaco (parte mais interna na aplicação da porcelana). Após esta cobertura, para a fabricação do corpo do disco, foi realizada a aplicação da porcelana, feita em pequenas camadas de aproximadamente $2 \mathrm{~mm}$ cada, em todo o seu perímetro circular.

Após a aplicação de cada camada da porcelana (conforme mostra a figura 1) o disco foi levado ao forno para a queima. O tempo de espera para o resfriamento da peça foi de aproximadamente uma hora e meia, para que só então uma nova camada pudesse ser aplicada. Esta operação foi repetida até que o disco ficasse com um diâmetro maior do que $26 \mathrm{~mm}$, necessário para a posterior usinagem (retificação).
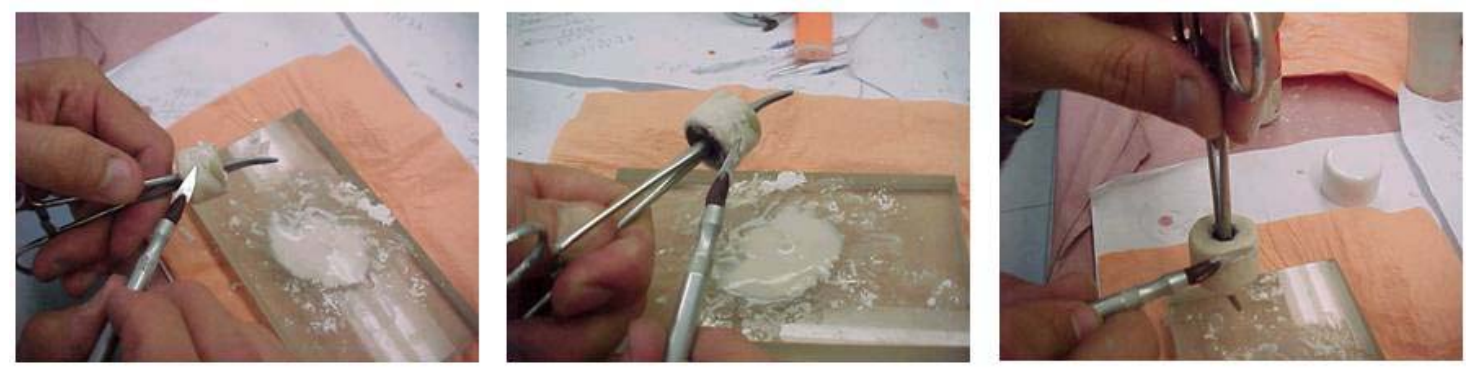

Figura 1: Aplicação da porcelana na preparação do disco dinâmico.

A porcelana utilizada foi a Duceram (produzida pela empresa alemã Degussa ${ }^{\circledR}$ ) da cor C4. A temperatura de coquição do opaco foi de $970{ }^{\circ} \mathrm{C}$ e a temperatura de coquição do corpo do disco foi de $950{ }^{\circ} \mathrm{C}$. A padronização da porcelana por cor e temperaturas de coquição é importante uma vez que não se sabe de antemão se diferentes porcelanas ou cores de uma mesma porcelana podem apresentar, depois de retificadas, topografias diferentes, parâmetro de extrema importância para o estudo da agressividade.

Interno ao anel da liga metálica foi introduzido uma bucha de nylon permitindo o preciso encaixe do disco ao eixo do banco de ensaios. Posteriormente à introdução da porcelana o disco foi retificado (como é visto na figura 2). Para esta operação utilizou-se uma retificadora tangencial plana com um cabeçote posicionado sobre sua mesa que permitiu a retificação da superfície cilíndrica. Utilizou-se um rebolo diamantado fabricado com ligante resinóide, granulometria de 126 mesh, especificação 4664 - B B126, com $350 \mathrm{~mm}$ de diâmetro externo, $128 \mathrm{~mm}$ de diâmetro interno e $10 \mathrm{~mm}$ de largura de corte, fabricado pela empresa Master do Brasil ${ }^{\circledR}$. 


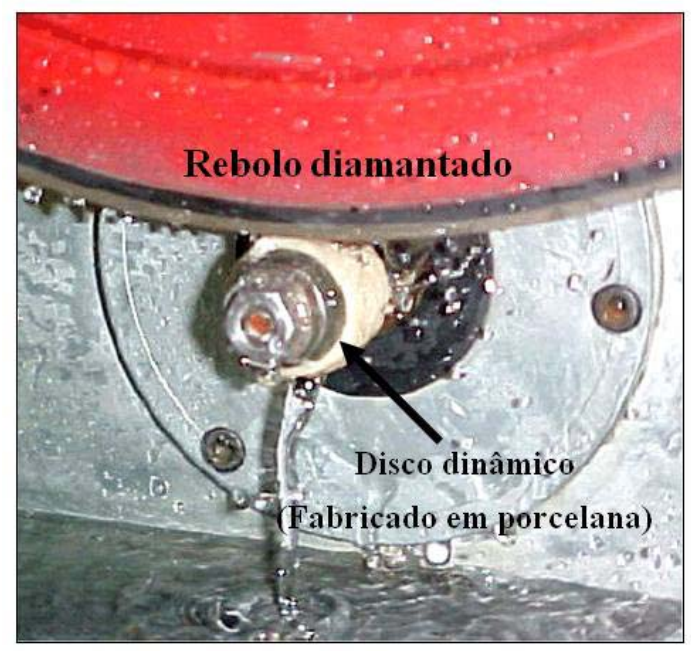

Figura 2: Detalhe da retificação do disco de porcelana. Água foi usada para a refrigeração do processo.

$\mathrm{Na}$ retificação a velocidade de corte empregada no rebolo foi de $33 \mathrm{~m} / \mathrm{s}$ e a rotação foram aferidas com o emprego de um tacômetro manual.

No processo de retificação realizado o sentido de giro dos discos foi discordante. Água foi utilizada para a refrigeração. A rotação do disco de porcelana proporcionou uma velocidade tangencial constante de 18 $\mathrm{m} / \mathrm{s}$. Estas velocidades foram utilizadas para a obtenção de um melhor acabamento superficial na porcelana, tendo em vista a expectativa de que com o aumento da velocidade consiga-se obter uma melhor qualidade superficial.

\subsection{Disco Estático}

Os discos estáticos ou discos agredidos são os recobertos com resina. Para sua confecção foram fabricados dezoito anéis de aço com baixo teor de carbono (ABNT 1020). A superfície circular externa destes anéis foi recartilhada para garantir uma melhor fixação das resinas e suas laterais foram retificadas, para assegurar que todos tivessem a mesma espessura e que as faces ficassem paralelas entre si.

Utilizou-se um processo de eletro-deposição de zinco para evitar a oxidação dos discos e a identificação dos mesmos foi feita por meio de marcação (com punção) de um código de letras.

Para facilitar e melhorar o recobrimento do disco com resina foi realizada uma matriz constituída por um parafuso central, duas arruelas metálicas, e duas arruelas lisas de teflon confeccionado com o diâmetro interno igual ao do parafuso e diâmetro externo de $26 \mathrm{~mm}$ (figura 3). Após o encaixe do disco na matriz foi feita a aplicação da resina sobre o mesmo com o auxílio de espátulas odontológicas, seguida de imediata polimerização de uma fina camada na superfície recartilhada, em uma extensão de $8 \mathrm{~mm}$ (correspondente à largura da ponta do aparelho fotopolimerizador), a qual representa cerca de 1/9 do perímetro circular da matriz.

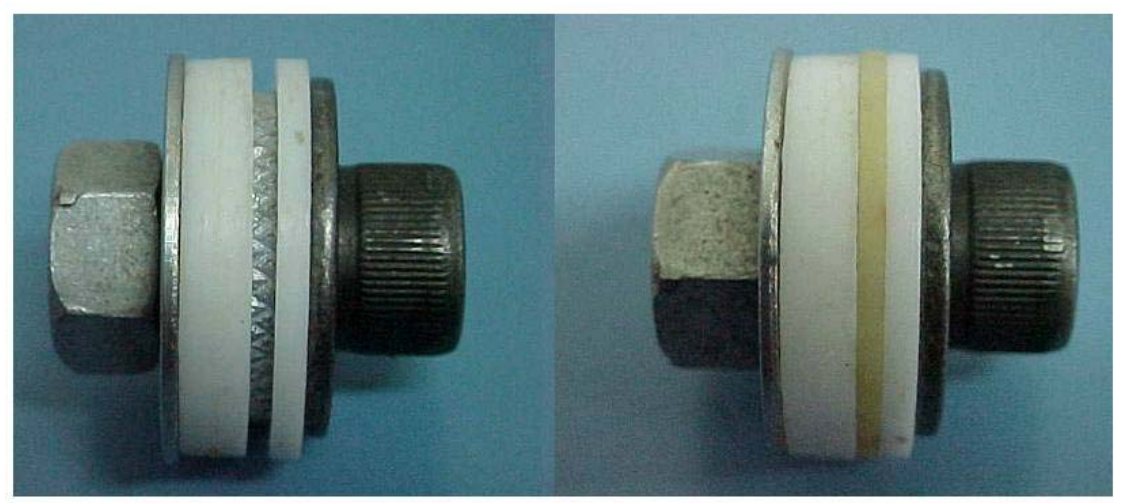

Figura 3: Matriz de teflon usada na confecção dos discos de resina. 
$\mathrm{Na}$ preparação dos discos estáticos diversos cuidados foram tomados. A ponta do aparelho foi encostada na resina para a execução da polimerização, separada apenas por uma fita de poliéster de uso odontológico, para evitar que a mesma grudasse na ponta do aparelho. Este procedimento foi realizado para que fosse mantida uma distância constante de polimerização, a fim de evitar variações na qualidade da mesma.

O aparelho fotopolimerizador utilizado foi o CL - K50, da empresa Kondortech ${ }^{\circledR}$. Neste aparelho a irradiação da fonte luminosa é feita através de uma lâmpada halógena. Este aparelho também foi aferido previamente à confecção de cada corpo de prova, considerando como satisfatória uma intensidade luminosa acima de $300 \mathrm{~mW} / \mathrm{cm}^{2}$. O radiômetro utilizado é da marca Demetron.Todas as resinas utilizadas foram da cor A2.

O processo de recobrimento descrito foi repetido até que todo o disco estivesse recoberto. Finalizada a sua cobertura com a resina, os anéis foram imediatamente imersos em água destilada deionizada. Após isso, aguardava-se um tempo de $24 \mathrm{~h}$ antes de realizar a sua retificação para a obtenção do diâmetro final. Para todas as amostras o tempo de pós-polimerização foi o mesmo, ou seja, o tempo esperado, após a cura da resina, antes de submetê-la a esforços a fim de desgastá-la foi o mesmo. Toda esta etapa de recobrimento do disco de aço com resina foi realizada em laboratório com controle ambiental de temperatura e de umidade. A temperatura ambiente foi mantida entre 23 e $24^{\circ} \mathrm{C}$ e a umidade relativa do ar ficou entre 40 e $60 \%$, mantida por um aparelho desumidificador.

A retificação para o ajuste do diâmetro final foi feita com a ajuda do mesmo cabeçote citado na fabricação do disco de porcelana. Para a retificação utilizou-se um rebolo de óxido de alumínio $\left(\mathrm{Al}_{2} \mathrm{O}_{3}\right)$ branco, com diâmetro externo de $350 \mathrm{~mm}$, diâmetro interno de $76,2 \mathrm{~mm}$, espessura de $50 \mathrm{~mm}$, granulometria 120 mesh e mantido a uma velocidade de corte constante de $33 \mathrm{~m} / \mathrm{s}$.

$\mathrm{O}$ cabeçote da retificadora foi deslocado transversalmente em relação ao disco e o rebolo foi progressivamente deslocado para baixo, executando um "mergulho" no interior da resina (de cerca de 5 a 10 $\mu \mathrm{m}$ de cada vez), até que o diâmetro externo desta ficasse com 24,00 mm. Deve-se destacar que os diâmetros dos discos dinâmico e estático devem ser os mesmos, devido à imposição do desenvolvimento do modelo matemático.

\subsection{Método de Avaliação do Desgaste das Resinas Compostas}

O método consiste em manter o disco de resina posicionado exatamente acima do disco de porcelana, havendo no início do ensaio apenas um contato pontual entre eles. Para que isso ocorra o disco de porcelana, que será dotado de giro para a realização do ensaio, é preso na ponta do eixo de um cabeçote posicionado sobre a mesa da retificadora. Acima deste conjunto está o disco de resina, preso a uma haste de deslocamento vertical do banco de ensaios. Esta haste tem o objetivo de não permitir qualquer outro tipo de deslocamento do disco que não seja o vertical, ocorra à medida que o disco de porcelana girante desgaste a resina. Sobre esta haste ainda é colocada uma carga, que atua como uma força vertical constante para impulsionar o disco de resina estático contra o disco de porcelana girante. $\mathrm{O}$ banco de ensaios pode ser visto na figura 4. 


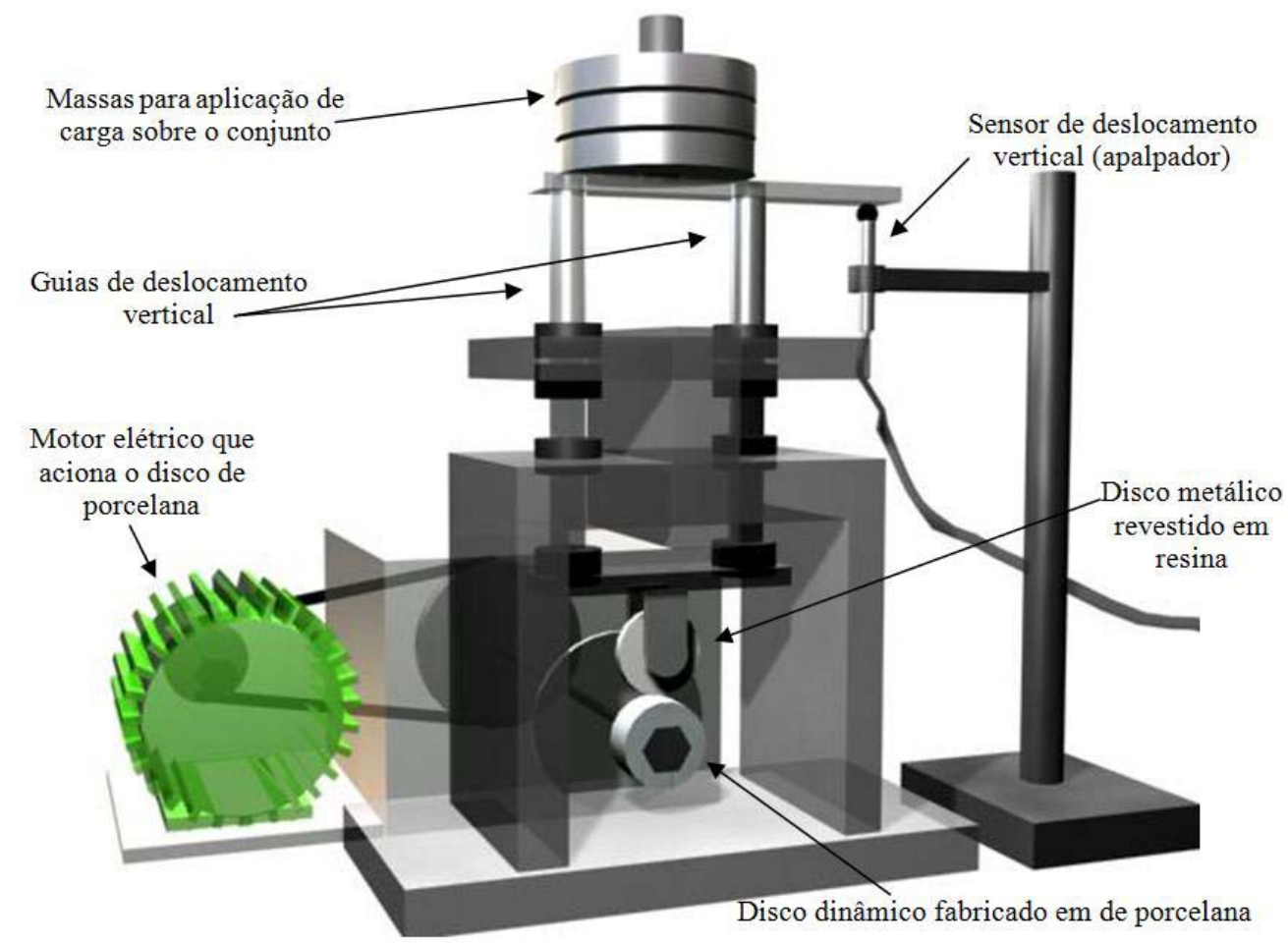

Figura 4: Desenho representativo do banco de ensaios utilizado.

Os parâmetros usados nos ensaios foram: velocidade de corte de $18 \mathrm{~m} / \mathrm{s}$, sob uma força normal constante de $16 \mathrm{~N}$, escolhidos através de ensaios preliminares.

Utilizando um medidor eletrônico com precisão micrométrica (da marca TESATRONIC ${ }^{\circledR}$ - modelo TT60, com um apalpador modelo 32.10904), mediu-se o deslocamento vertical do disco fixo (d) à medida que o mesmo ocorreu devido ao desgaste da resina. $\mathrm{O}$ apalpador foi posicionado na extremidade de uma haste vertical.

O medidor eletrônico foi conectado a uma placa especial para a aquisição de dados, usando como elemento de ligação uma caixa de conectores. Este aparato tem a função de registrar os sinais de tensão enviados pelo aparelho. Para isso foi desenvolvido um programa específico, utilizando o software LabView ${ }^{\circledR}$. Este programa registra os dados de tensão enviados pelo aparelho à placa e os armazena em um arquivo. Além disso, ele também gera um gráfico da tensão para que os operadores do sistema se certifiquem de que o ensaio está sendo realizado de forma satisfatória.

Após terem sido coletados os sinais de tensão são transformados em deslocamento, dado em micrometros. Para isso, é usada uma equação de calibração previamente encontrada. Um gráfico é construído onde no eixo vertical são registrados os valores do deslocamento, em $\mu \mathrm{m}$, e no eixo horizontal, o tempo gasto para a aquisição dos dados elevado à $3 / 2$.

Segundo Coelho [13] , este processo permite obter uma reta em que o coeficiente angular da mesma fornece o parâmetro " $\mathrm{a}_{1}$ " utilizado no cálculo da agressividade, que de acordo com um desenvolvimento matemático adequado à estrutura do banco de ensaios, resulta na equação (1).

$$
k=\frac{2 b \sqrt{4 r}}{3 F_{n}}\left(a_{1}\right)^{3 / 2}
$$

Onde $\mathrm{k}\left[\mathrm{mm}^{3} / \mathrm{N} . \mathrm{s}\right]$ representa a agressividade de uma resina (do disco estático) contra a porcelana (do disco dinâmico); Fn [N] a força normal necessária para efetuar-se a remoção de material num determinado tempo; $b$ [mm] a largura dos discos fixos; $r$ [mm] o raio dos discos; e $a_{1}$ o coeficiente angular da reta de regressão linear.

O valor do parâmetro k encontrado na equação foi utilizado nas comparações feitas neste trabalho. 


\section{RESULTADOS E DISCUSSÕES}

Para cada resina e para cada tempo de polimerização foi feito um número de repetições de cinco ensaios. No total foram testadas seis marcas de resinas, há três tempos diferentes de polimerização para cada uma. As resinas utilizadas foram: Charisma, Fill Magic, Suprafill, Tetric Ceram, XRV Herculite e Z100. Os tempos de polimerização foram de $10 \mathrm{~s}, 20 \mathrm{se} 40 \mathrm{~s}$.

Os resultados obtidos estão apresentados na tabela 1. Esta tabela mostra os cinco valores de agressividade k encontrados para cada resina, a média e o desvio padrão abreviado por Desv. Pad.

Tabela 1: Valores de agressividade, em $\mathrm{mm}^{3} / \mathrm{N}$.s, encontrados com os resultados laboratoriais.

$\begin{array}{cccccccc} & 10 \mathrm{~s} & 20 \mathrm{~s} & 40 \mathrm{~s} & & 10 \mathrm{~s} & 20 \mathrm{~s} & 40 \mathrm{~s} \\ & 0,061967 & 0,058982 & 0,055228 & & 0,10997 & 0,099897 & 0,088306 \\ & 0,060445 & 0,051735 & 0,048312 & & 0,10403 & 0,095718 & 0,090311 \\ \text { Charisma } & 0,060283 & 0,067585 & 0,050229 & \text { Tetric } & 0,10757 & 0,10353 & 0,093301 \\ & 0,061116 & 0,052271 & 0,058858 & \text { Ceram } & 0,097224 & 0,099926 & 0,086216 \\ & 0,061967 & 0,058982 & 0,055228 & & 0,10997 & 0,099897 & 0,088306 \\ \text { Média } & 0,061156 & 0,057911 & 0,053571 & \text { Média } & 0,105753 & 0,099794 & 0,089288 \\ \text { Desv. Pad } & 0,000804 & 0,006439 & 0,004251 & \text { Desv. Pad } & 0,005352 & 0,002766 & 0,00267 \\ & 0,067487 & 0,075691 & 0,076096 & & 0,097077 & 0,07553 & 0,081562 \\ & 0,072467 & 0,074622 & 0,070689 & & 0,092667 & 0,079439 & 0,087537 \\ \text { Fill Magic } & 0,073422 & 0,077297 & 0,07873 & \text { Xrv_Herculite } & 0,092086 & 0,074154 & 0,086983 \\ & 0,068075 & 0,078413 & 0,075869 & & 0,093621 & 0,079145 & 0,084132 \\ & 0,067487 & 0,075691 & 0,076096 & & 0,097077 & 0,07553 & 0,081562 \\ \text { Média } & 0,069788 & 0,076343 & 0,075496 & \text { Média } & 0,094506 & 0,07676 & 0,084355 \\ \text { Desv. Pad } & 0,002911 & 0,001501 & 0,002934 & \text { Desv. Pad } & 0,002411 & 0,002381 & 0,002858 \\ & 0,078178 & 0,074966 & 0,064172 & & 0,12363 & 0,13451 & 0,069455 \\ & 0,076199 & 0,071958 & 0,061295 & & 0,122287 & 0,13248 & 0,063867 \\ \text { Suprafill } & 0,076247 & 0,066732 & 0,066903 & \text { Z100 } & 0,12296 & 0,11818 & 0,063794 \\ & 0,074494 & 0,070449 & 0,062702 & & 0,10802 & 0,13715 & 0,061025 \\ & 0,078178 & 0,074966 & 0,064172 & & 0,12363 & 0,13451 & 0,069455 \\ \text { Média } & 0,076659 & 0,071814 & 0,063849 & \text { Média } & 0,120105 & 0,131366 & 0,065519 \\ \text { Desv. Pad } & 0,001556 & 0,003449 & 0,002084 & \text { Desv. Pad } & 0,006779 & 0,007555 & 0,003771\end{array}$

Para melhor visualização, a partir dos valores apresentados na tabela 1 foi elaborada a figura 5 que mostra os valores médios de agressividade e o desvio padrão para cada condição. 


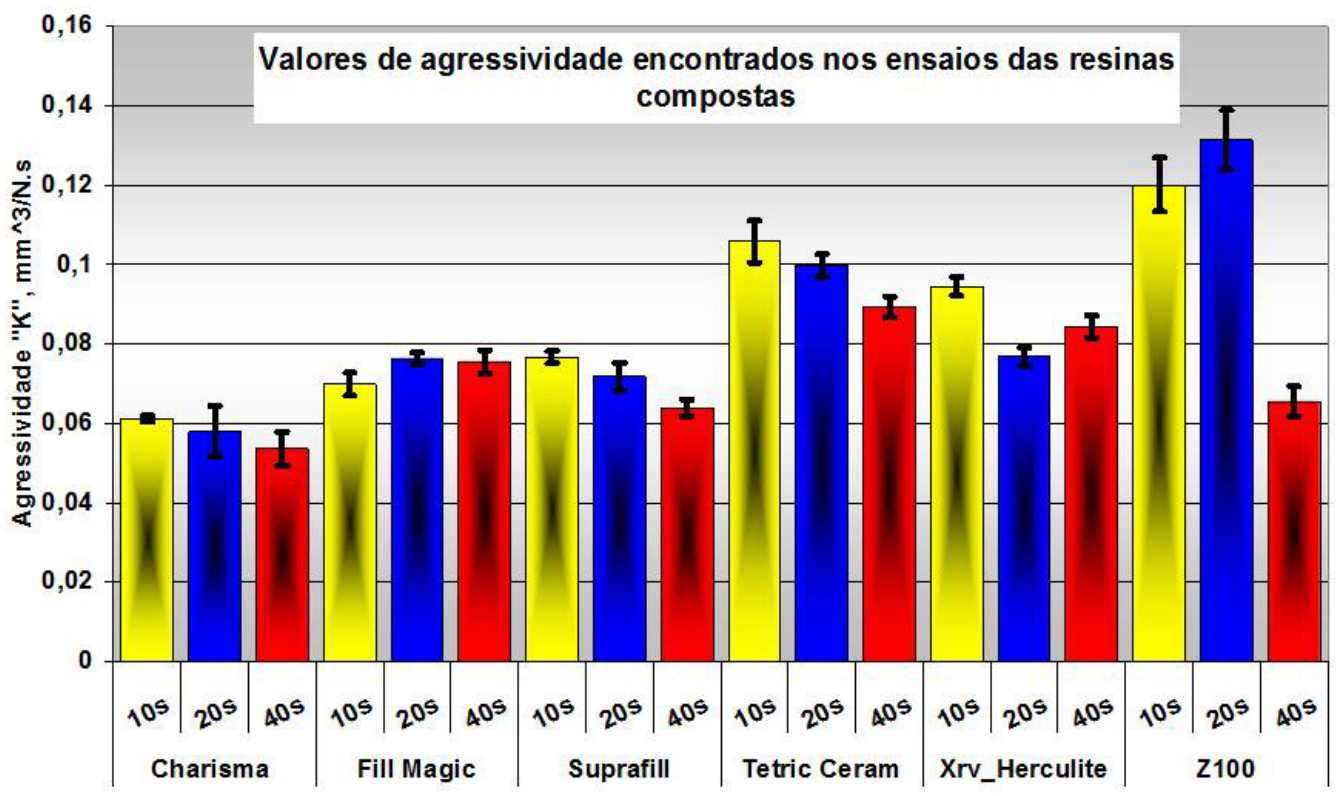

Figura 5: Resultados de agressividade obtida nos ensaios com resinas.

Estes valores foram submetidos à análise estatística pelo método ANOVA ou análise de variância. A análise foi feita a dois critérios de classificação, divididos quanto às resinas e quanto ao tempo de polimerização, a um nível de significância de $5 \%$.

A comparação entre materiais mostra que a resina Charisma, polimerizada no tempo de 40 s obteve o melhor desempenho nos testes aplicados. Os piores desempenhos foram obtidos na resina Z100, com tempos de polimerização iguais a 10 s e 20 s.

Os resultados mostram diferentes comportamentos das resinas quanto ao tempo de polimerização. Para a resina Charisma, a análise estatística demonstrou melhora na resistência da resina obtida para o tempo de 40s, não havendo diferenças entre os tempos de 10s e 20s. O mesmo ocorreu para a resina Tetric Ceram e Z100, sendo que a última foi quem mais demonstrou diferenças entre sua resistência ao desgaste abrasivo com a variação de tempos. Para este material, a agressividade encontrada no ensaio com a resina polimerizada por um tempo de 40 s foi próximo a $100 \%$ inferior ao valor obtido com o tempo de 20 s.

Para a resina Fill Magic não foram observadas diferenças estatísticas entre os tempos de polimerização.

A resina Suprafill, apresentou melhoras gradativas com o aumento do tempo de polimerização. A análise mostra melhor resistência para 40s de polimerização, seguido de 20 s e finalmente pelo tempo de 10 s.

Para a resina XRV Herculite o tempo de melhor desempenho foi 20 s seguido de 40 s e finalmente 10 s.

A Tabela 2 resume os resultados apresentados e permite a comparação com os valores de tempo de polimerização sugeridos, em bula, pelos fabricantes.

A comparação dos resultados mostra que quatro da seis resinas estudadas apresentaram seu melhor desempenho nos tempos de polimerização sugeridos por seus fabricantes, contudo isso apenas demonstra acerto quantitativo dos tempos. Uma análise continuada capaz qualificar estes resultados em aceitáveis ou não para aplicação em pacientes é imprescindível.

A alta repetibilidade apresentada pelo sistema, vista através dos baixos valores de desvio padrão encontrados para os cinco ensaios realizados em cada material e condição de fotopolimerização, confirma sua sensibilidade ao estudo do desgaste abrasivo das resinas compostas.

Contudo ainda é restrita a aplicabilidade dos resultados na qualificação dos materiais para uso em seres humanos. Sugere- se para continuidade do trabalho a escolha de um corpo padrão, de material bastante homogêneo, capaz de criar uma escala padrão para o ensaios, que permita, comparar valores em função desta escala, e em conjunto com ensaios laboratoriais, qualificar estes resultados em níveis aceitáveis, ou não aceitáveis, de materiais, aparelhos e condições de utilização. 
Tabela 2: Resumo dos resultados de desempenho dos materiais de acordo com tempo de polimerização e comparação com os valores sugeridos pelos fabricantes.

\begin{tabular}{|c|c|c|c|c|}
\hline Resinas & $\begin{array}{c}\text { Tempo de } \\
\text { polimerização } \\
\text { sugerido pelo } \\
\text { fabricante }\end{array}$ & $\begin{array}{c}\text { Tempo de } \\
\text { melhor } \\
\text { desempenho }\end{array}$ & $\begin{array}{c}\text { Tempo de } \\
\text { desempenho } \\
\text { intermediário }\end{array}$ & $\begin{array}{l}\text { Tempo de pior } \\
\text { desempenho }\end{array}$ \\
\hline Charisma & $20 \mathrm{~s}$ & $40 \mathrm{~s}$ & \multicolumn{2}{|c|}{ 10s e 20s (estatisticamente iguais) } \\
\hline Fill Magic & $\begin{array}{c}20 \text { s ou até que a } \\
\text { superfície se apresente } \\
\text { lustrosa e brilhante }\end{array}$ & \multicolumn{3}{|c|}{ Não foram encontradas diferenças estatísticas } \\
\hline Suprafill & $20 \mathrm{~s}$ & $40 \mathrm{~s}$ & $20 \mathrm{~s}$ & $10 \mathrm{~s}$ \\
\hline Tetric Ceram & $40 \mathrm{~s}$ & $40 \mathrm{~s}$ & \multicolumn{2}{|c|}{ 10s e 20s (estatisticamente iguais) } \\
\hline XRV Herculite & $20 \mathrm{~s}$ & $20 \mathrm{~s}$ & $40 \mathrm{~s}$ & $10 \mathrm{~s}$ \\
\hline $\mathrm{Z} 100$ & $40 \mathrm{~s}$ & $40 \mathrm{~s}$ & \multicolumn{2}{|c|}{ 10s e 20 s (estatisticamente iguais) } \\
\hline
\end{tabular}

\section{CONCLUSÕES}

A partir dos dados obtidos nos ensaios experimentais concluiu-se que:

O banco de ensaios mostrou elevada sensibilidade e capacidade ao estudo dos diferentes tempos de polimerização em resinas compostas de aplicação odontológica. A boa repetibilidade obtida nos ensaios, permitiu baixos valores de desvio padrão dos resultados, permitindo que fossem constatadas diferenças estatísticas entre as condições analisadas.

As resinas estudadas apresentaram resultados variados aos tempos de polimerização. Quatro entre seis resinas estudadas, Charisma, Suprafill, Tetric Ceram e Z100, tiveram os melhores resultados de resistências ao desgaste abrasivo quando foram fotopolimerizadas no tempo de 40s, correspondente ao maior tempo utilizado nos ensaios.

Em um dos materiais do estudo, a resina Fill Magic, não foram encontradas diferenças estatísticas entre os tempos de polimerização, e na resina XRV Herculite, o tempo de 20s foi que apresentou os melhores resultados.

Estes dados comprovam a importância da aplicação de testes a estes materiais anterior à sua comercialização, a fim de conhecer suas características de desempenho geral e específicas maximizando suas características.

\section{AGRADECIMENTOS}

Os autores agradecem à FAPESP pelo apoio financeiro concedido para esta pesquisa.

\section{BIBLIOGRAFIA}

[1] AMERICAN DENTAL ASSOCIATION, Council on Dental Materials, Instruments, and Equipment, "Obstacles to the Development of a Standard for Posterior Composite Resins", Journal of the American Dental Association, v. 118, n. 5, pp. 649-651, May. 1989.

[2] Miranda, A.C.C., PALUdeTti, F.C., CARVAlho, H.A., MATSON, E., CARVAlHO, S.A., "Resina posterior: Uma avaliação ético-clínica". Revista do Instituto Ciência da Saúde, v. 2, n. 1, pp. 11-6, jan./jun., 1990.

[3] WENDT, S.L., LEINFELDER, K.F., "Clinical evaluation of posterior resin composite: 3-year results", American Journal of Dentistry, v. 7, n. 4, pp. 207-221, 1994.

[4] COLLINS, C.J., BRYANT, R.W., HODGE, L.V., "A clinical evaluation of posterior composite resin restorations: 8-year finding", Department of Clinical Dentistry, Wetstmead, Hospital Dental Clinical Scholl, Westmead, Austrália. 1997.

[5] MAIR, L.H., STOLARSKI, T.A., VOWLES, R.W., LLOYD, C.H., "Wear: mechanisms, manifestations and measurement. Report of a workshop", Journal of Dental Research, v. 24, pp. 141-148, 1996. 
[6] YAP, A.U.J., THEO, S.H., HASTINGS, G.W., LO, C.S., "Comparative wear ranking of dental restorative materials utilizing different wear simulation modes", Journal of Oral Rehabilitation, v. 24, pp. 574-580, 1997.

[7] CONDON, J.R., FERRACANI, J.L., "In vitro wear of composite with varied cure, filler level, and filler treatment”, Journal of Dental Research, v. 76, n. 7, pp. 1405-1411, Jan. 1997.

[8] MOMOI, Y., HIROSAKI, K., KOHNO, A., McCABE, J.F., Dental Materials, v. 13, pp. 82-88, march, 1997.

[9] VENHOVEN, B.A.M., DE GEE, A.J., DAVIDSON, C.L., DAVIDSON, W., Influence of filler parameters on the mechanical coherence of dental restorative resin composites, Department of Dental Materials Science, Academic Centre for Dentistry Amsterdam (ACTA), Louwesweg 1, 1066 E. A. Amsterdam, Netherlands, 1995.

[10] VIEIRA, G.F., FREIRE, I.A., AGRA, C.M., "Análise da irradiação de diversos aparelhos fotopolimerizadores", Revista de Odontologia da Universidade de São Paulo, v. 12, n. 4, pp. 395399, 1998.

[11] ABATE, P.F., ZAHRA, V.N., MACCHI, R.L., "Effect of photopolymerization variables on composite hardness", Journal of Prosthetic Dentistry, v. 86, n. 6, pp. 632-635, dec. 2001.

[12] HALVORSON, R.H., ERICKSON, R.L., DAVIDSON, C.L., "Energy dependent polymerization of resin-based composite", Dental Materials, v. 18, n. 6, pp. 463-469, sept. 2002.

[13] COELHO, R.T., Estudo Experimental da Propriedade de Dressagem de Rebolos na Retificação de Precisão Usando o Método do Disco Retificado, Dissertação de M.Sc., Escola de Engenharia de São Carlos-EESC-USP, São Carlos, SP, Brasil, 1991.

[14] DICKINSON, G.L., GERBO, L.R., LEINFELDER, K.F., 1993, “Clinical evaluation of a highly wear resistant composite", American Journal of Dentistry, v. 6, n. 2, pp. 85-7.

[15] KURACHI, C., TUBOY, A.M., MAGALHAES, D.V., BAGNATO, V.S., "Hardness evaluation of a dental composite polymerized with experimental LED-based devices", Dental Materials, v. 17, Issue 4, pp. 309-315, 2001.

[16] TSAI, P.C.L., MEYERS, I.A., WALSH, L.J., "Depth of cure and surface microhardness of composite resin cured with blue LED curing lights", Dental Materials, v. 20, Issue 4, pp. 364-369, 2004.

[17] CARVALHO JUNIOR, O.B., Avaliação "in vitro" da dureza superficial, da Contração de polimerização e da rugosidade de 29 resinas compostas, Tese de Doutorado, Universidade de São Paulo, Brasil, 2002. 